\title{
Multidisciplinary information application for structuring design
}

\section{Ensici, Ayhan}

Istanbul Technical University, Turkey. ayhan.ensici@khas.edu.tr

\begin{abstract}
Designers in teams continuously discuss information connected to design ideas, problems or solutions that whey developed in problem solving process. However some moments are more relevant for the process, thereby decisive for the final design. The "Critical situation method" (Badke-Schaub and Frankberger, 2003) which was developed in order to evaluate the data of the design work distinguishing between critical situations and routine work have been used to focus on those moments. Critical situations determine choice points with importance for the subsequent design process and the result. To track the information behavior patterns of the design team members provoking crucial design decision we concentrated critical situations of the types 'goal analysis, goal decision' and 'solutionsearch, solution-analysis and, solution-decision'
\end{abstract}

Keywords: Design Process, Information, Multidisciplinary Design Teams

\section{Introduction}

In response to changing markets, increasing global competition, and technological developments, companies strive to create successful products. The role of the designer has changed over the last years; in current industrial practice the most of the designers work as part of a team. The creation of design often happens with the cooperation among experts from various disciplines due to require wide heterogeneous information. Throughout the design process stages information processing in a multidisciplinary context is integral part of any design process. Information is shared or not shared, is applied or not applied among design team members. Thus, how this information processing takes place, has become increasingly vital to the overall success of product design.

During product development designers have to make countless decisions by utilizing multidisciplinary information. Design decisions constitute critical situations in the product development process (BadkeSchaub and Frankenberger, 1999) in the sense that they have a tremendous impact on the quality of the design solution. Thus, information used as part of the critical situations is one of the most important issues that influence the quality of the design decisions/outcome.

The design process can be seen as an information process where the design space of the problem will be gradually transformed into a space of solutions (Restrepo, 2004). Design problem solving is a process of 
application of information to construct the design solution space and which can be seen as a web of all possible solutions linked with operators presenting problem-solving operations. Generally application of design information determines the structuring of the design problem and the defining of the solution space. During design process multidisciplinary team members generate and share large amounts of information and knowledge in multiple context. Information shared by the teammates in critical situations has a decisive role on the direction of design process.

This paper aims to 1) investigate the application of multidisciplinary information and 2) explore the process of structuring design solutions in Critical Situations (Badke-Schaub \& Frankenberger, 1999).

\section{Design team studies}

\subsection{Information Behavior in Design Teams}

The number of studies aimed at understanding how designers design in teams has been increasing. In one of the first studies on design teams Tang and Leifer (1988) empirically investigated small group design sessions to understand collaborative workspace activity. The first systematic studies about design teams were presented in a session at the ICED93 conference. In 1994, the seminal Delft Protocols Workshop (Cross et. al., 1996) brought a number of researchers with an interest in design research together to apply different forms of protocol analysis on a common dataset. Team design aspects includes group aspects such as communication (Stemple and Badke-Schaub, 2002, Carrisoza and Seppard, 2000, Chiu M, 2002), collaboration (Kalay 2001), and interaction (Brereton at. al.1996).

\subsection{Aspects of Information in Critical Situations}

Critical situations are defined as 'turning points' with an important influence on the further direction of the design process and the product (Badke-Schaub and Gehrlicher, 2003). We derived from the types of critical situations regarding their aim in the problem solving process, such as goal analysis, goal-decision, solution-search, solution-analysis and solution decision.

Goal Analysis and Goal Decision: These two categories combine analysis and clarification of requirements, that means questioning and generating the demands and wishes of the given problem

Solution Search: This is the creation and generation of solutions and is mostly interconnected with the analysis of solutions

Solution Analysis and Decisions: These two categories refer to situations where the evaluation and selection of solutions take place (Badke-Schaub and Frankenberger, 2002)

\section{Experimental studies of design teams}

In order to observe and record multidisciplinary information aspects in design teamwork, it was decided to set up a group design task in a laboratory environment. The controlled settings in laboratory enable the observer to reduce the impact of context variables and allows to repeat the study. The group work was recorded, transcribed and then categorized. Using a group rather than an individual denoted that verbal articulations occurred in an unconditioned contrary to thinking-aloud protocols in an individual laboratory setting. 
Two design teams were analyzed. Three members for each of the teams were selected from different disciplines, working in the same company. Professional experts, consisting the design teams, had comparable background and experience of professional life.

The design task given to design teams has been considered to be able to be completed in assigned time. After briefing the experimental set-up of the study by researcher, participants had time to work on the assignment individually. The assignment was to design a portable brazier (barbecue) specific for the Turkish market that can be used at outdoor during picnic attractions in recreational green areas. The design task was common and is stated as follows:

"Design a portable brazier (barbecue) specific for the local market that can be used at outdoor during picnic attractions in recreational green areas. The company wants differentiation from other products in the market with design, innovation, quality and price in order to ensure a prestigious penetration to the market."

The research outlines the analysis of information behavior to gain a better understanding of multidisciplinary information activities in design teams and the relationship between information and structuring design solution. In order to observe and record the application of information in design team work, two multidisciplinary design teams were analyzed, each consisting of three professional experts from different disciplines. The design teams' working processes, in a laboratory environment that has been set up, were recorded, transcribed and then categorized. For deeper understanding of the process the verbal transcripts have been categorized and coded according to coding schemes (a) information behavior and (b) information content. Latter one used to explore the complexity of the design process and to be able to code information behavior activities more precisely.

\subsection{Method of Analysis}

In this study, protocol analysis was used as method for analyzing the structure the information behavior of design teams. Protocol analysis is accepted as a efficient approach for investigating the human cognitive processes and it has been widely used to gain insight into cognitive processes of individuals as well as teams (Cross et al., 1996, Dorst, 1997; Valkenburg, 2000; Stemple and Badke-Schaub, 2002). Interview and document analysis were also used for the data collection together with protocol analysis method.

Applying Protocol Analysis Method enables the researcher to investigate the verbal records which reflects the mental processes. Teamwork situations are especially helpful as every verbal expression presents the own mental model which between members is a separate communication message. Thus every message is part of cognitive activities of information processing in order to develop the design as the goal of the task. Members in a design team talk about different issues and generate more than one explanation in different contexts, often even in one expression. Every meaningful piece of verbal expressions, one or more consecutive sentences, a word or even an exclamation indicates a cognitive action. In our analysis each expression may consist of one, two or more meaningful segments which are accepted as a cognitive action.

Protocol analysis depends on the segmentation of the transcription into meaningful pieces. Primarily verbal expression of the members are transcribed. Every package of message including a cognitive action or reasoning accepted as segmented part of protocol. Although, recognizing segments requires sociological aspects to be considered, mostly recognizing the segments including design information or a cognitive action in design process is easy for a design expert.

The main idea of our method is separating the transcribed design process of team to routine situations on one hand and critical situations on the other. 
One of the most crucial steps in protocol analysis is the development of a coding system. The categories of a coding system should be theoretically consistent and exhaustive to ensure that every information behavior can be assigned to one category. In this research the coding schemes were developed; (a) information behavior, (b) critical situations and (c) design content. Latter one used to explore the whole design process and to be able to code information behavior activities more precisely. Information behavior categories formulated based on previous empirical studies, on theoretical models in cognitive psychology and on design methodology, and then tested and further refined based on data from the experiment.

Documents produced by the design team during the design process have been utilized to detect the design issues the team discussed. Additionally semi-structured interviews and questionnaires were conducted. Design content is different from design issues due to design issues are specific topics related to given design task. Design teams continuously discussed issues coming from design ideas, problems or solutions that whey developed in problem solving process. Those issues could be discussed in the context of one content, or as mostly happened, they were discussed in the context of multiple design contents.

The critical situations have been detected among information behavior of team to trace the multidisciplinary design information by considering their contexts. The context of the information determines the influence of that information in a design solution

\section{Results}

Although design team members have to apply different information to structure the design problem, generate and develop solutions, and evaluating design solutions, often actual design activity does not progress in this direction. In design team, situations of disagreement, conflict or misunderstanding between designers arises and generally flaw ensues. Hence design solutions are not only based on rational cognitive processes with utilizing needed information (Cross and Cross, 1996).

The results gained from two case studies, one was according to defined criteria evaluated as successful design process and the other one as unsuccessful, have been compared. The analysis of the data explore different information behavior during the design process and show patterns of information application within the critical situations. The results of the research confirm

- that designers are not necessarily aware of the presence of different multidisciplinary information through design team work,

- that designers can make decisions with insufficient information or

- that designers can't reach decisions because of redundant information generation.

This research is one attempt to provide a foundation for describing design solving patterns by means of information processing behavior in critical situations of decision making. 


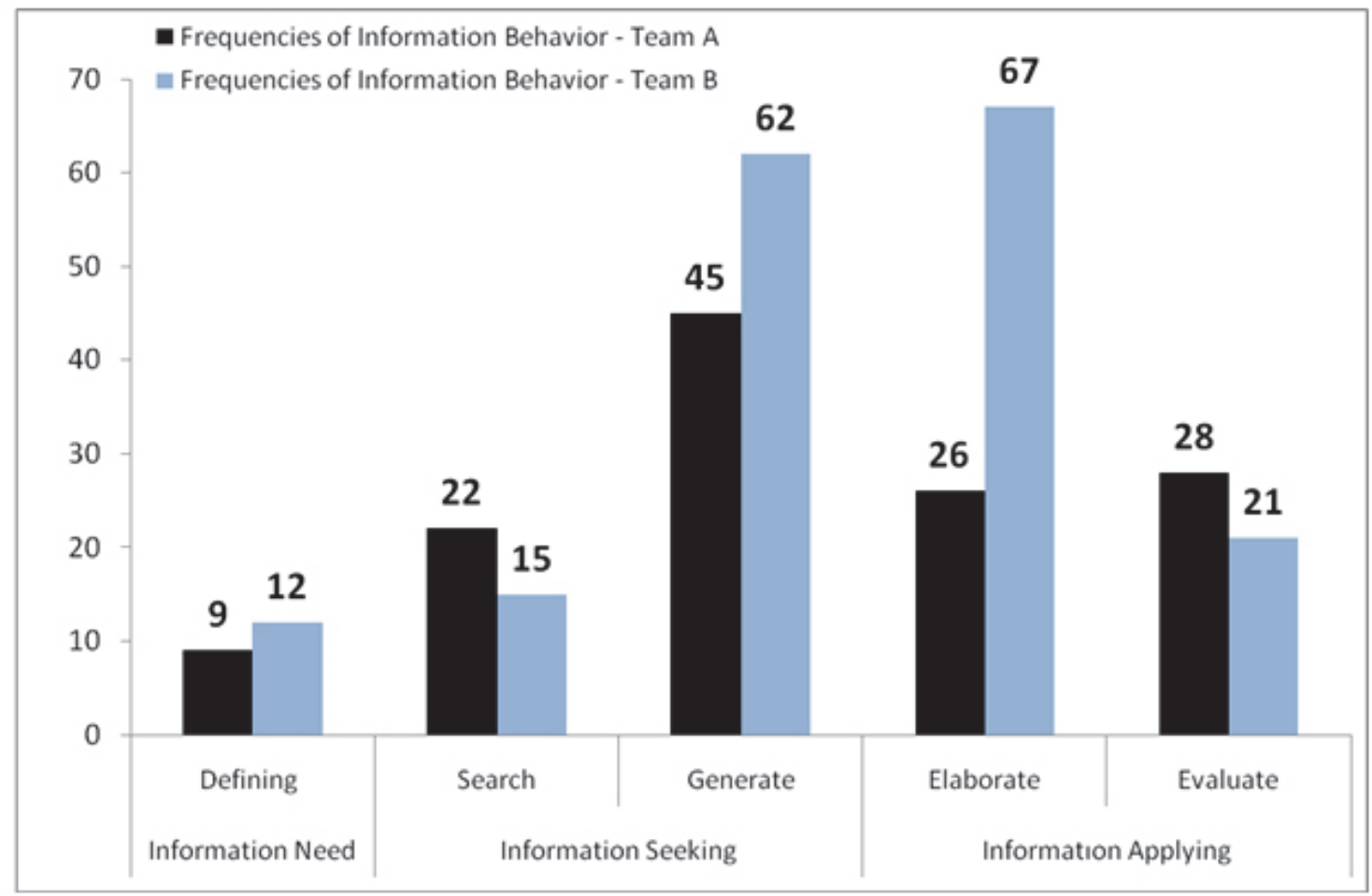

Fig. 1 Information Behavior Frequencies of Team A and Team B

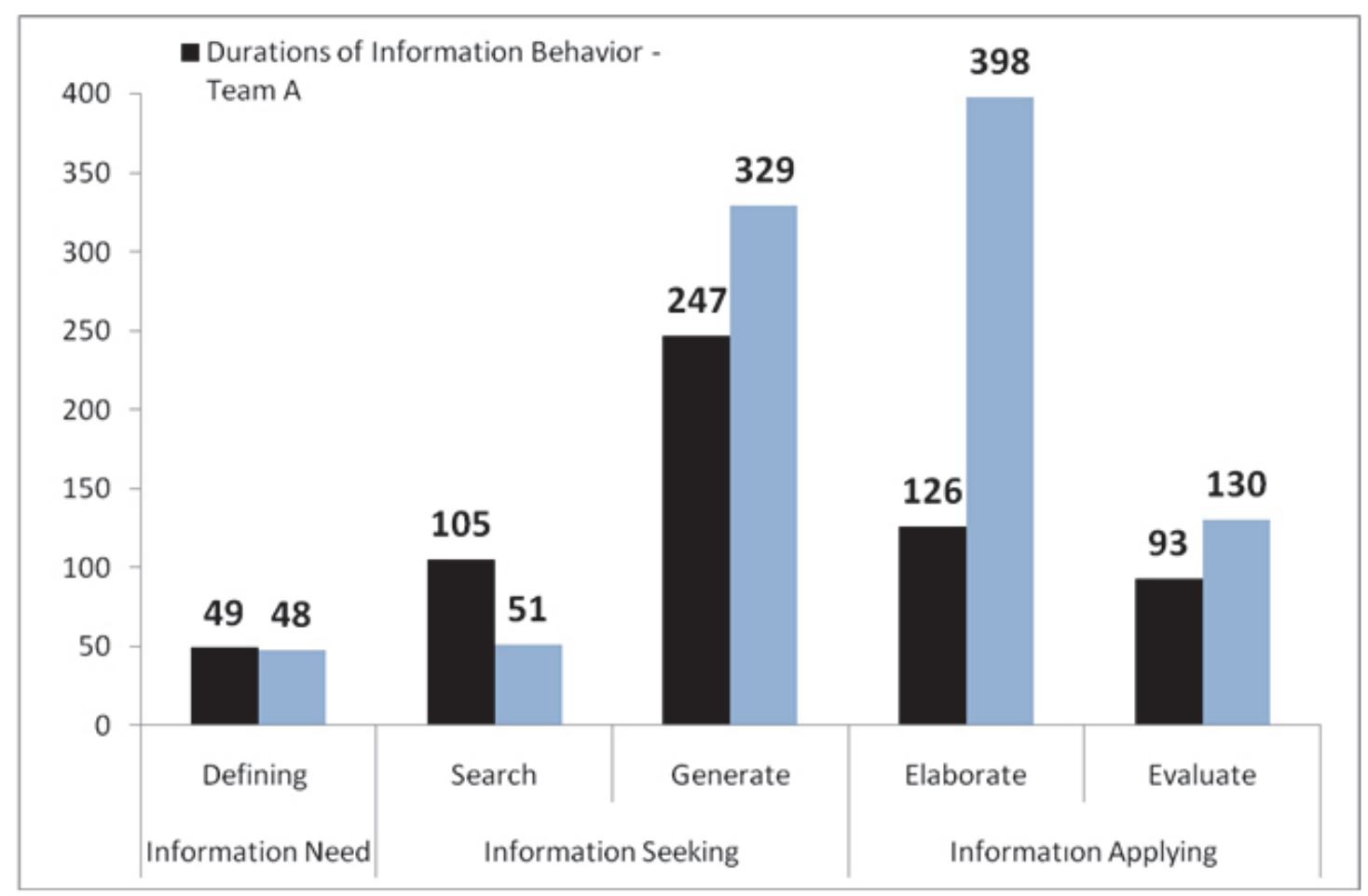

Fig. 2 Information Behavior Durations of Team A and Team B 


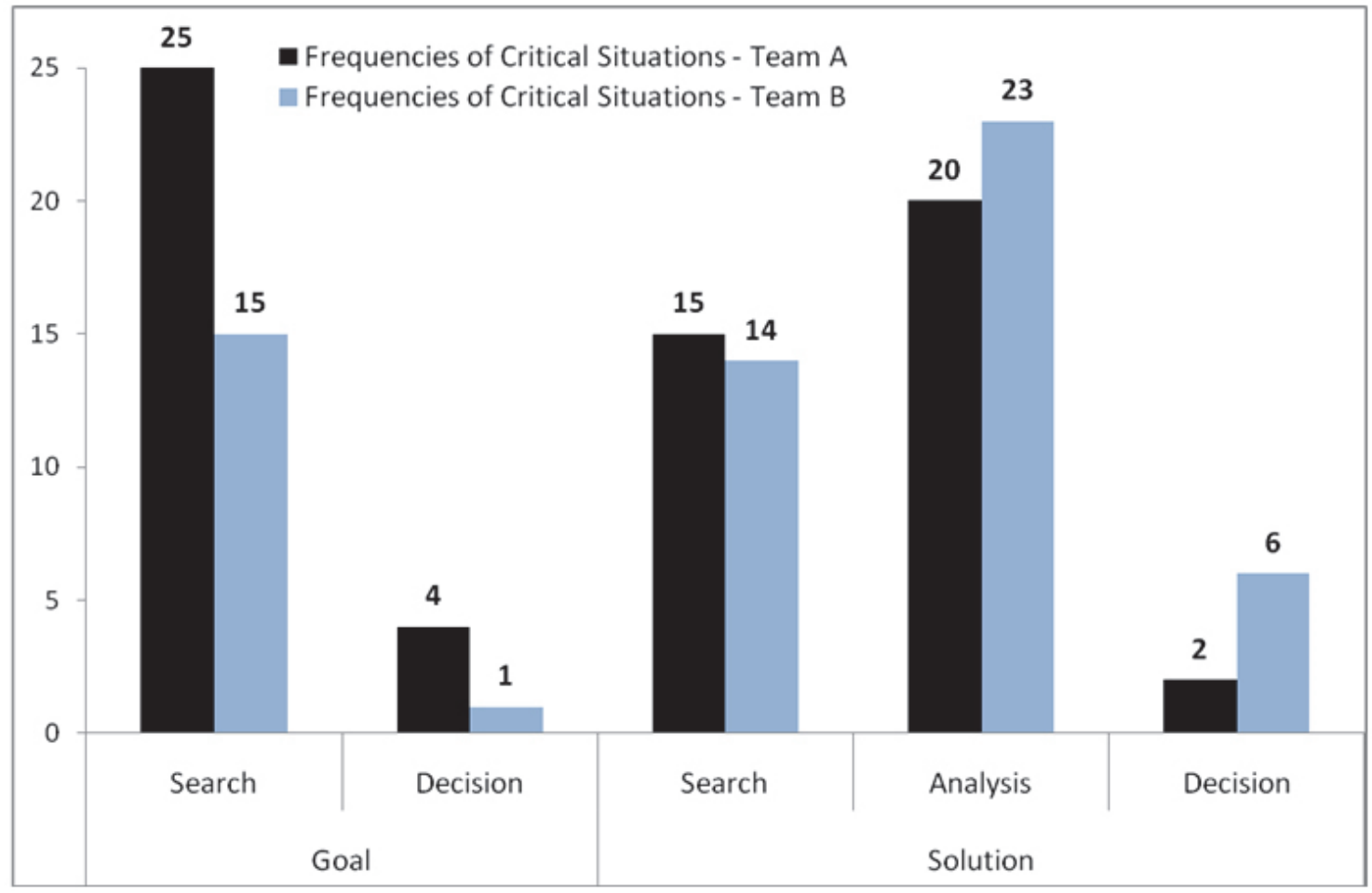

Fig. 3 Frequencies of Critical Situations occurred within Information Behavior: Team A and Team B

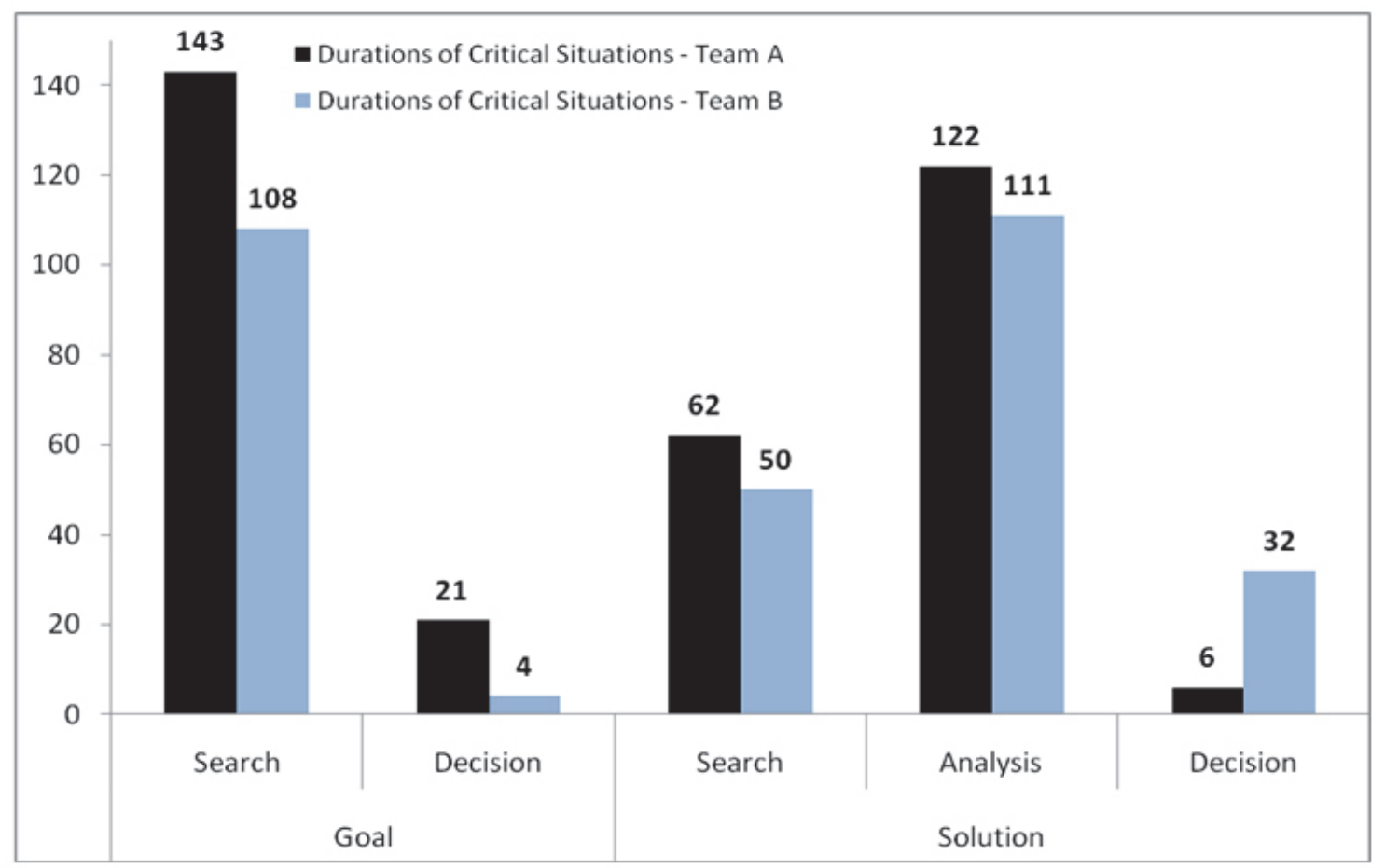

Fig. 4 Durations of Critical Situations occurred within Information Behavior: Team A and Team B 


\section{References}

BADKE-SCHAUB, P. \& FRANKENBERGER, E., (1999). Analysis of design projects. Design Studies, 20, 481-494.

BADKE-SCHAUB, P. \& FRANKENBERGER, E., (2002) Analysing and Modelling Cooperative Design by the Critical Situation Method, Le Travail Humain, 65, Number 4, pp. 293-314

BADKE-SCHAUB, FRANKENBERGER, E., (2003), Design Representation in Critical Situations of Product Development, Design Representation, Gabriela Goldschmidt and William L. Porter (Eds.), Springer-Verlag London, pp. $105-126$

BADKE-SCHAUB, GEHRLICHER, A., (2003), Patterns of Decisions in Design: Leaps, Loops, Cycles, Sequences and Meta-processes, ICED 11 proceedings, Stockholm

CROSS, N. \& CROSS, A., (1996), Observations of Teamwork and Social Processes in Design. In N. Cross, H. Christiaans, \& K. Dorst (Eds.), Analysing Design Activity (pp. 291-317). Chichester: John Wiley \& Sons.

RESTREPO, J., (2004) Information Processing in Design. ISBN 90-407-2552-7 Delft University Press. Delft, The Netherlands 